\title{
La comprensión lectora en el idioma inglés y su influencia en el aprendizaje significativo
}

Reading comprehension in english language and its influence in the meaningful learning

Isabel Grimanesa Olín Zegarra

Universidad Nacional de San Agustín. Arequipa Perú.

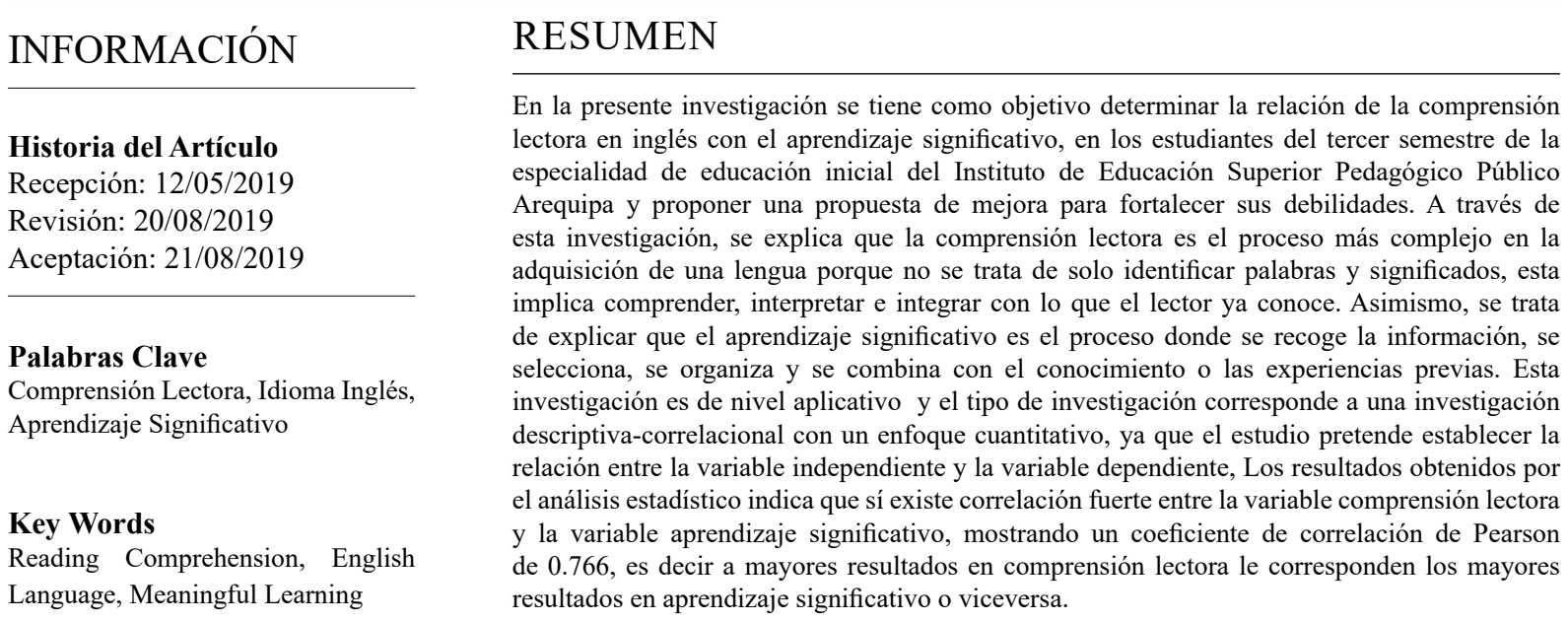

DOI

https://doi.org/10.35286/veritas. v20i2.236

\begin{abstract}
The main objective of the present research is to determine the relationship of reading comprehension in English with meaningful learning, among students from the third semester of the specialty of Initial Education from the Institute of Higher Education Pedagogical Public Arequipa and to present a proposal to enhance and to strengthen their weaknesses. Through this research it is explained that reading comprehension is a more complex process in a second language because it is not just about identifying words and meanings, in English language teaching, this skill is very important because it implies understanding and interpreting a with what the reader knows. Likewise, it is about explaining that meaningful learning is the process where information could be obtained, selected, organized and combined with knowledge or previous experiences.This research is of application level and the research type corresponds to a descriptive-correlational investigation with a quantitative approach, since the study aims to establish the relationship between the independent variable and the dependent variable, The results obtained by the statistical analysis indicate that there is a strong correlation between the reading comprehension variable and the significant learning variable, showing a Pearson correlation coefficient of 0.766 , that is, higher results in reading comprehension correspond to the greater results in meaningful learning. or vice versa.
\end{abstract}

\section{INTRODUCCIÓN}

Hoy en día la sociedad peruana del siglo XXI se enmarca en un contexto mundial cada vez más competitivo, por ende, la educación tiene la ardua y difícil tarea de enfrentar las exigencias del mundo globalizado donde se desarrollan los estudiantes de hoy y los obliga a estar al día en diversos temas de interés personal, profesional, tecnológicos, sociales, culturales, entre otros. Es importante mencionar que todos estos conocimientos solo se obtienen a través de la lectura y una óptima comprensión, lo que indica que, mientras más y mejor lean nuestros estudiantes, mejor preparados estarán, estimando que la lectura es la base de la enseñanza. De acuerdo a este contexto, dos aspectos resultan ser relevantes para mi objeto de estudio: En primer lugar, la comprensión lectora

Correspondencia:

Isabel Grimanesa Olín Zegarra

isa021161@hotmail.com en el idioma inglés, que forma parte de una problemática inherente en los diversos programas curriculares donde las consecuencias por la deficiencia en la comprensión de textos afectan de manera directa en la formación académica y posteriormente, en la baja calidad de profesionales. Viendo esto desde otro punto de vista, se opina que este problema nace en la educación básica, prueba de ello son los últimos resultados del PISA, 2015 (Programme for International Student Assessment), en donde el Perú ocupó el puesto 63 de 69 naciones evaluadas en comprensión lectora (PISA, 2015). En segundo lugar, el aprendizaje significativo también se convierte como parte importante de esta investigación por su relación en el proceso activo de analizar los conocimientos nuevos con los conocimientos previos, para que tengan una gran significatividad sustantiva y no arbitraria, según lo manifestado por Ausubel (2012).

Muchas son las definiciones que existen entre estos dos temas de gran importancia en la educación superior. 
La comprensión lectora juega un rol importante en el aprendizaje del estudiante y en su rendimiento académico, así como en su éxito profesional. Un buen desarrollo de esta habilidad implica un buen aprendizaje significativo para el futuro profesional de educación superior porque le permite acceder libre, rápida y eficientemente a todo tipo de información bibliográfica de acuerdo a su especialidad, sobre todo si se trata de textos en lengua extranjera. Solé (1992), establece varias relaciones entre la lectura y las acciones de comprender y de aprender, para esta autora no puede haber una lectura significativa sin comprensión y tampoco puede haber una lectura significativa que no fomente nuevos aprendizajes, También, analiza que a partir de la lectura de un texto, se puede fomentar un aprendizaje significativo gracias a la comprensión lectora. Es decir, que lo que el estudiante logre comprender o entender va a depender de gran manera de los conocimientos previos. Desde mi propia experiencia analizo que la lectura y comprensión deberían tener un grado alto de concreción pedagógica para solucionar problemas dentro del aprendizaje del idioma inglés en el estudiante de educación superior. En el área de inglés también se desarrolla la capacidad específica: comprensión de textos (Reading Comprehension); que busca la recepción de la información para una adecuada interacción comunicativa y lograr de este modo nuevos aprendizajes (Paya, 2018).

Como docente del área de idioma extranjero inglés he observado las dificultades que presentan los futuros docentes del tercer semestre de educación inicial del Instituto de Educacion Superior Pedagógico Público Arequipa, cuando leen un texto que no está en su idioma nativo, demostrando de esta manera debilidad en su comprensión y al no entender el significado del texto, este no se podrá relacionar con los conocimientos previos, por lo tanto, no logran un aprendizaje significativo. Por otro lado, presentan un bajo nivel de destrezas en el uso del idioma, demostrando que luego de haber estudiado inglés en la educación básica; ellos no han adquirido los conocimientos y destrezas necesarios para su comprensión, ello se puede deber que en el proceso de enseñanza del idioma se ha considerado a docentes de otras especialidades con la finalidad de completar su horario de trabajo, generando que docentes improvisados, sin o con bajo conocimiento solo se enfoquen en la enseñanza de la gramática olvidándose que el estudiante necesita desarrollar las habilidades linguísticas como son el aprender a leer, a escribir, a comunicarse, a comprender y entender el idioma inglés; el proporcionar y estimular el desarrollo de tales habilidades debe ser el centro de nuestra práctica docente, como profesores de inglés dando énfasis en el desarrollo de la habilidad lectora a fín de obtener aprendizajes más significativos. En el proceso de enseñanza-aprendizaje del idioma inglés dos componentes son importantes: el docente y el estudiante, sin dejar de lado la metodología, el currículo, y el contexto educativo. Una buena combinación de estos componentes en condiciones favorables pueden llevar a los estudiantes a desarrollar mejores niveles de aprendizaje, pero en circunstancias desfavorables pueden interferir y afectar los resultados grandemente (Harmer, 2001).

Diversos estudios sobre como la comprensión lectora influye en el aprendizaje significativo realizados en diferentes instituciones tanto nacionales como internacionales han recibido la atención de múltiples investigadores al evaluar el grado de relación que existe entre estos dos temas de gran importancia en la educación como es el caso de Andino (2015), en su investigación: "Estudio de comprensión lectora y su influencia en el aprendizaje significativo de los alumnos de 6to año paralelo "A" de educación básica de la Unidad Educativa Brethren" en la Maestría en Ciencias de la Educación de la Pontificia Universidad Católica del Ecuador; en una de sus conclusiones menciona que la comprensión lectora incide en el aprendizaje significativo al tratarse de una destreza básica para el entendimiento de los contenidos y en el caso de Odalis Lilian Malásquez Quispe y Roberta Yisela Malásquez Quispe (2014) en la tesis titulada: La Comprensión Lectora y el Aprendizaje significativo de los estudiantes del 5to grado de la I.E N 7240 de Villa El Salvador. 2014, concluyen que la comprensión lectora y el aprendizaje significativo son dos procesos fundamentales porque están relacionados con las propias experiencias, saberes previos y con las circunstancias cotidianas del estudiante que le permitirá enfrentar con criticidad diversos momentos de su vida, demostrándose en ambos casos que existe una estrecha relación entre la comprensión lectora y el aprendizaje significativo.

Finalmente, en el presente trabajo de investigación se ha logrado comprobar que si existe una relación significativa ente la compresión lectora en el idioma inglés con el aprendizaje significativo, descriptivamente hablando, indica una correlación positiva fuerte.

\section{MATERIAL Y MÉTODOS}

El enfoque de la investigación es cuantitativa, el nivel es aplicado de tipo descriptivo-correlacional (Sánchez \& Reyes, 2009) no experimental, de corte transversal. La población está conformada por 70 estudiantes matriculados en el tercer semestre de la especialidad de educación inicial en el año 2018 del Instituto de Educación Superior Pedagógico Público Arequipa, No se trabajó con muestra por lo que la población no es muy extensa. Para la realización de todo el análisis estadístico tanto de las variables, la verificación de la hipótesis, el análisis de la $\mathrm{R}$ de Pearson y la confiabilidad del instrumento se utilizó el programa IBM SPSS v23. Para calcular el coeficiente de correlación entre la variable comprensión lectora y la variable aprendizaje significativo, se utilizó el coeficiente de correlación de Pearson,

El instrumento seleccionado fue un cuestionario considerando el concepto de Hernández, Fernández, and Baptista (2014) tanto para la variable independiente comprensión lectora en el idioma inglés con un total de 27 ítems y el aprendizaje significativo con un total de 20 ítem utilizando para ambos cuestionarios la escala de respuestas en formato (Likert, 1969): nunca, pocas veces, algunas veces, generalmente y siempre con una calificación de 1 a 5 donde 1 es "nunca" y 5 "siempre". Asimismo, para determinar la confiabilidad de los instrumentos se utilizó el Alfa de Cronbach a través del programa IBM SPSS v23 utilizando una muestra aleatoria de 10 estudiantes. 


\section{RESULTADOS}

Los resultados del Alfa de Cronbach para el cuestionario de comprensión lectora en el idioma inglés presentó un valor mayor a $0.8(0.810)$, demostrando una confiabilidad buena del instrumento. De igual modo el Alfa de Cronbach para el cuestionario aprendizaje significativo fue mayor a 0.9 (0.939), demostrando una confiabilidad excelente del instrumento. Se utilizó como criterio general (George \& Mallery, 2003) para validar estos resultados.

Respecto al resultado del coeficiente de correlación entre la variable comprensión lectora y la variable aprendizaje significativo se encontró un coeficiente de correlación $\mathrm{R}$ de Rearson de 0.766.

Tabla 1: Cálculo del coeficiente de correlación entre la variable comprensión lectora y la variable aprendizaje significativo Fuente: Elaboración propia - Spss v23

\begin{tabular}{|c|c|c|c|}
\hline & & $\begin{array}{l}\text { COMPRENSIÓN } \\
\text { LECTORA }\end{array}$ & $\begin{array}{l}\text { APRENDIZAJE } \\
\text { SIGNIFICATIVO }\end{array}$ \\
\hline \multirow{4}{*}{$\begin{array}{l}\text { COMPRENSIÓN } \\
\text { LECTORA }\end{array}$} & $\begin{array}{l}\text { Correlación R } \\
\text { de Pearson }\end{array}$ & 1 &, $766^{* *}$ \\
\hline & & & \\
\hline & Sig. (bilateral) & & ,000 \\
\hline & $\mathrm{N}$ & 70 & 70 \\
\hline \multirow{3}{*}{$\begin{array}{l}\text { APRENDIZAJE } \\
\text { SIGNIFICATIVO }\end{array}$} & $\begin{array}{l}\text { Correlación R } \\
\text { de Pearson }\end{array}$ &, $766^{* *}$ & 1 \\
\hline & Sig. (bilateral) & ,000 & \\
\hline & $\mathrm{N}$ & 70 & 70 \\
\hline
\end{tabular}

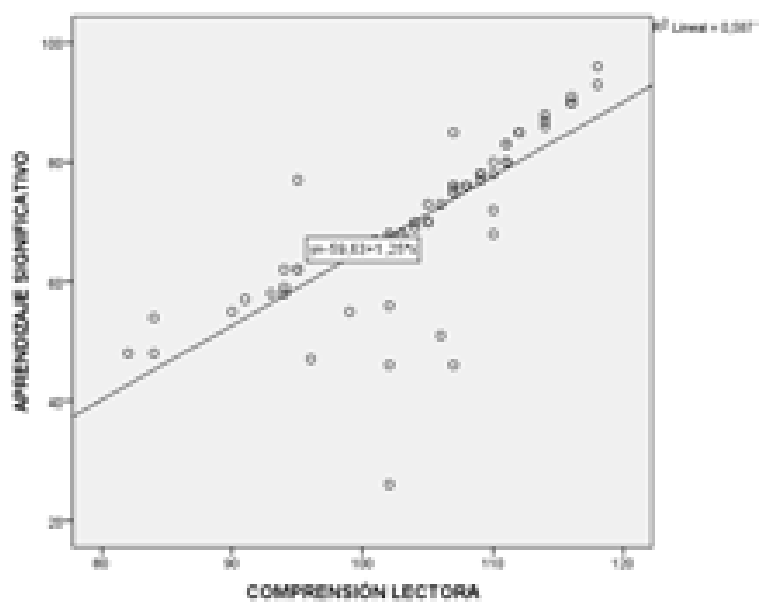

Fig. 1: Diagrama de dispersión entre comprensión lectora y aprendizaje significativo

Al observar la tabla 1, el p-valor o significancia bilateral 0.000 , es menor que el nivel de significancia de $\alpha 0.05$; por lo cual se entiende que existe relación entre ambas variables.

Al tratarse de dos variables cuantitativas, el COEFICIENTE DE CORRELACIÓN R DE PEARSON 0.766 lo que indica una CORRELACIÓN POSITIVA FUERTE entre la variable comprensión lectora y la variable aprendizaje significativo.

Al ver el gráfico 1, diagrama de dispersión, se observa que la relación entre ambas variables es directamente proporcional, es decir a mayores resultados en comprensión lectora le corresponden los mayores resultados en aprendizaje significativo, y a menores resultados en comprensión lectora le corresponde menores resultados en aprendizaje significativo. La ecuación $\mathrm{y}=-59.63+1.25 \mathrm{x}$ nos muestra la relación entre dichas variables, además el valor R2 lineal que es el COEFICIENTE DE DETERMINACIÓN, cuyo valor es 0.587 , nos indica que dicha ecuación obtenida por regresión lineal representa el $58.7 \%$ de los casos donde esta relación se cumple.

\section{DISCUSIÓN}

En la presente investigación denominada la comprensión lectora en el idioma inglés y su influencia en el aprendizaje significativo se ha demostrado que sí existe una relación significativa entre ambas variables, con un nivel de correlación Pearson de $\mathrm{r}=0.766$ la cual es una relación POSITIVA FUERTE; por ende, se acepta la hipótesis alterna y se rechaza la nula. Esta información se puede corroborar tomando en cuenta los antecedentes que se hacen mención, donde diversos autores en diferentes tesis analizadas concluyen que existe una relación significativa entre la comprensión lectora y el aprendizaje significativo, y comparando con los resultados de la presente investigación, se puede afirmar que cuando hay un buen nivel de comprensión lectora se logra un buen aprendizaje significativo, deduciendo así que existe una relación entre estas dos variables estudiadas.

Se concluye, según los análisis y resultados encontrados, que la comprensión lectora es un componente esencial para la adquisición de una segunda lengua, en este caso el idioma inglés, por lo que un idioma no consiste únicamente de la palabra hablada sino también de la palabra escrita. El desarrollo de esta habilidad favorece a que el estudiante sea capaz de interpretar diversos textos y párrafos en este idioma, asimismo favorece el rendimiento académico del estudiante de educación superior, aquel estudiante que lee y comprende lo leído por ende tiene un buen aprendizaje significativo.

Se sugiere que los estudiantes y docentes del area muestren mayor interés y sostenibilidad por la comprensión lectora del idioma inglés junto a su proceso de aprendizaje significativo, pues la sociedad requiere de personas capaces de dinamizar, comprender, reflexionar, pensar en inglés, y emplear información a partir de estrategias y métodos eficaces de trabajo académico sobre la lengua extranjera, para emitir juicios de valor, utilizar y relacionarse con todo tipo de estructuras y constructos académicos, para satisfacer necesidades de la vida como ciudadanos reales, constructivos, comprometidos con la resolución de los problemas actuales y reflexivos.

\section{AGRADECIMIENTO}

Agradezco a la Universidad Nacional de San Agustín, a los docentes por su dedicación en la formación de grandes profesionales, porque cada uno de ellos, con sus valiosas aportaciones, me permitieron crecer como persona y como profesional. A la comunidad educativa del Instituto de Educación Pedagógico Público Arequipa por permitirme llevar a cabo este trabajo de investigación. Finalmente, a cada una de las personas que han permitido realizar esta tesis agradeciéndoles de corazón por todo el apoyo, colaboración, ánimo y sobre todo el cariño, amistad, paciencia y comprensión. 


\section{REFERENCIAS BIBLIOGRÁFICAS}

1. Andino, A. (2015). Estudio de comprensión lectora y su influencia en el aprendizaje significativo de los alumnos de sexto año paralelo "A" de educación básica de la unidad educativa Brethren. Tesis maestría.

2. Ausubel. (2012). The acquisition and retention of knowledge: A cognitive view: Springer Science \& Business Media.

3. George, D., \& Mallery, P. (2003). Using SPSS for Windows step by step: a simple guide and reference (4th ed. ed.).

4. Harmer, J. (2001). The practice of English language teaching. London/New York.

5. Hernández, S. R., Fernández, C. C., \& Baptista, L. P. (2014). Metodología de la investigación (Vol. 3): México: McGraw-Hill.

6. Likert, R. (1969). El factor humano en la empresa: su dirección y valoración. Bilbao.Devito.

7. Paya, L. C. (2018). Estrategias cognitivas en la comprension de ingles-lectura en estudiantes universitarios. Educere, 22(72), 375-386.

8. PISA. (2015). PISA Learning for Tomorrow's World: First Results from PISA 2003: Simon and Schuster.

9. Sánchez, H., \& Reyes, C. (2009). Metodología y diseños en la investigación científica.

10. Solé, I. (1992). Estrategias de lectura (Vol. 137): Graó. 